\title{
Risk Factors of Neonatal Death in Neonatal Intensive Care Unit at Sheik Hassan Yabare Referral Hospital, East Ethiopia, 2019: Unmatched Case Control Study
}

\author{
Mohamed Omar Osman*, Abdikani Mawlid Nur, Abdilahi Omer Abdilahi, \\ Ahmed Mohammed Ibrahim
}

Department of Public Health, Jigjiga University, Jigjiga, Ethiopia

Email address:

Mahamadwehliye@gmail.com (M. O. Osman),Wehliye@jju.edu.et (M. O. Osman)

${ }^{*}$ Corresponding author

To cite this article:

Mohamed Omar Osman, Abdikani Mawlid Nur, Abdilahi Omer Abdilahi, Ahmed Mohammed Ibrahim. Risk Factors of Neonatal Death in Neonatal Intensive Care Unit at Sheik Hassan Yabare Referral Hospital, East Ethiopia, 2019: Unmatched Case Control Study. American Journal of Pediatrics. Vol. 6, No. 2, 2020, pp. 162-167. doi: 10.11648/j.ajp.20200602.28

Received: March 7, 2020; Accepted: March 23, 2020; Published: April 23, 2020

\begin{abstract}
Child and neonatal mortality are a grave problem in Ethiopia. The first one month of life-the neonatal period is the most vulnerable time for a child's survival. Ethiopia remains to have one of the highest rates of neonatal deaths in Africa with the rate of 30 deaths per 1000 live births. Therefore, the aim of this study was to determine the risk factors of neonatal death among Neonates admitted in Neonatal Intensive Care Unit at Sheik Hassan Yabare Referral Hospital, East Ethiopia. Materials and methods: Unmatched Case Control Study was conducted from August 1 to August 30, 2019. Data were extracted from the medical records of neonates admitted during a two-year period from July 30, 2017 to August 01, 2019. using pretested questionnaire. Cases and controls were selected using the systematic sampling technique. Data were entered using Epidata 3.1 and exported to SPSS version 23.0 for cleaning and analyses. Bivariate and multivariate logistic regressions were done to determine factors associated with neonatal mortality and P-values $<0.05$ were considered statistically significant. Results: A total of $76(33.3 \%)$ cases and $152(66.7 \%)$ controls were included in this study. The multivariable logistic regression analysis showed that the possible risk factors of neonatal death in this study were; history of maternal urinary tract infection or sexually transmitted infection [AOR=3.058; 95\% CI (1.136-8.229)]. Conclusion: The major risk factor of neonatal mortality in this study was maternal urinary tract infection, it was also investigated risk factors such as Residence, Birth weight, Parity, Antenatal visits, Type of breastfeeding, premature rupture of membranes or Place of delivery, Intrauterine growth retardation but were not observed to be predictors for neonatal death. Consolidation of the existing risk-based prevention and treatment strategies as well as boosting up of antenatal care and institutional delivery practices are recommended to improve neonatal survival.
\end{abstract}

Keywords: Risk Factors, Neonatal Death, NICU, SHYRH, East Ethiopia

\section{Introduction}

Despite progress over the past quarter-century, millions of newborns, children and young adolescents die every year, mostly of preventable or treatable causes such as infectious diseases and injuries. The neonatal period, which is universally accepted as beginning at birth and ending at 28 completed days of life [1]. Neonatal death is defined as the death of a live born infant, irrespective of gestational age at birth, within the first 28 completed days of life [2]. Neonatal death can be typified into viable and non-viable deaths depending on the gestational age at which they were born, and where they were born [2]. Which can be further subdivided into early neonatal deaths (deaths between 0 and 7 completed days of birth) and late neonatal deaths (deaths after 7 days to 28 completed days of birth) [3].

It is important to address the main causes of neonatal mortality, which often vary from the causes of death for older children. According to United Nations Children's Fund (UNICEF) report in 2018 revealed that Preterm birth, 
intrapartum-related complications (birth asphyxia or problem of breathing at birth), infections and such as sepsis, meningitis and pneumonia were the main causes of newborn death [4].

According to different studies, there are many factors contributing to neonatal mortality; among these are educational level of the mother $[5,6]$. duration of pregnancy [7]. home delivery without skilled provider [8]. pregnancy complication [9]. pre-term birth $[10,11]$. Birth weight [12, 13]. Other studies disclosed that the neonatal mortality was associated with Social exclusion, maternal literacy, negative parental attitudes arising from social environment, gender bias, ability to pay, and lack of basic prenatal, natal and postnatal care are the main determinants of newborn survival in developing countries [14, 15].

Due to the use of advanced interventions to prevent and care in many parts of the world, the neonatal mortality has declined [16]. Weather Skilled care at birth, basic and comprehensive obstetric care, management of preterm births (including the use of antenatal corticosteroids) and newborn care practices such as neonatal resuscitation, immediate and exclusive breastfeeding, kangaroo mother care for low preterm neonates, contributed the reduction of neonatal mortality [17].

Immunization of pregnant women has been proven to be advantageous to the mother as well as the newborn by decreasing morbidity and mortality during this exposed period with the huge success of maternal tetanus vaccination for the prevention of neonatal tetanus [18].

Worldwide, 2.5 million children died in the first month of life. in 2018-approximately 7,000 neonatal deaths every day, with one third dying on the first day with huge discrepancies in the level of neonatal mortality persist across regions and countries [19]. The highest Neonatal death occurred in subSaharan Africa and South Asia, with each estimated at 28 and 26 deaths per 1,000 live births in 2018. A neonate born in sub-Saharan Africa or in South Asia is ten times and nine times more likely to die in the first month than a neonate born in a high-income country [19].

The targets set by the Lancet Commission on Investing in Health and the Sustainable Development Goals (SDGs) for decreasing neonatal deaths is defined as 12 neonatal deaths per 1,000 livebirths by 2030 [20].

Globally, the neonatal mortality rate fell by 51 per cent from 1990 to 2017), a lesser reduction in mortality than among children aged 1-59 months (63 per cent) [21].

According to the 2019 EDHS MINI results showed that the neonatal mortality rate (NMR) was 30 , deaths per 1,000 live births, which has remained stable compared the 2016 EDHS which was 29 deaths per 1,000 live births [22].

Many centers in this country have studied the risk factors of neonatal death with different designs. But these studies had investigated limited risk factors associated with neonatal death and in contrary there was no any study related to risk factors of neonatal mortality has been carried out in this region so far. Therefore, this study was carried out to determine risk factors associated with neonatal mortality among Neonates Admitted in the Neonatal Intensive Care Unit at Sheik Hassan Yabare Referral Hospital, East Ethiopia 2019.

\section{Methods and Materials}

\subsection{Study Design and Period}

A hospital based Retrospective Unmatched case control study design was conducted in NICU at Sheik Hassan Yabare Referral Hospital from August 1 to August 30, 2019.

\subsection{Study Area}

The study was conducted in Sheik Hassan Yabare Referral Hospital, Jigjiga East Ethiopia. Jigjiga town is located 626 $\mathrm{km}$ east from Addis Ababa, capital city of Ethiopia.

As of 2015 EFY Jigjiga city has a total population of 426,122 out of which 85,650 are in reproductive age group (15-49 years). The city has 30 sub-districts (smallest administration units) out of which 20 are urban and 10 are rural, most of the people are of ethnic Somalis (97\%) and Muslim (98\%) [23].

Sheik Hassan Yabare Referral Hospital (SHYRH) also known as Jigjiga university Sheik Hassan Yabare Referral Hospital was inaugurated in January, 2017. SHYRH is the largest and modern hospital, providing state-of-the-art, and wide-ranging service under one roof in eastern Ethiopia, the hospital has a total of 342 beds, Operation Theatre with the state of art facilities, a 13 bed Intensive Care Unit, emergency service, as well as Inpatient and Outpatient services, Pharmacy with Clinical Lab facilities are offered. Annually, roughly 2000 neonates obtain care each year.

\subsection{Source and Study Population}

\subsubsection{Source Population}

The source population for the study will be all neonates' who were admitted in Neonatal Intensive Care Unit at Sheik Hassan Yabare Referral Hospital with the specified period.

\subsubsection{Study Population}

All newborn $\leq 28$ days of life and who were admitted Neonatal Intensive Care and selected to include in the study. Cases were defined as all neonates who died within the first 28 days of life while controls were selected among those who survived the first 28 days of life. two controls were matched to each case based on the date of birth.

\subsection{Inclusion and Exclusion Criteria}

The inclusion criteria as a case was neonatal deaths occurred within the study period while neonates who survived within the study period were included as a control in the NICU and had complete file. Exclusion criterion for case and control groups was neonate's death and survival occurred within the study period in the NICU but having incomplete patient file. 


\subsection{Sample Size Determination}

A two-population proportion formula (using STATCAL application of Epi-Info) will be used to estimate the sample size required for the study by considering that the proportion of neonates with who's their Mode of delivery was cesarean section among the controls of $25.5 \%$ exposure, $95 \%$ CI, $80 \%$ power of the study control to case ratio of 1:2 to detect an odds ratio of 2.5 which was estimated from a study done by others [24]. Accordingly, by adding $10 \%$ for the nonresponse rate, 76 cases and 152 controls (a total sample size of 228 was the estimated sample size in this study. Cases and controls were selected using systematic random sampling.

\subsection{Data Collection Techniques and Tools}

Data was collected using structured questionnaire adapted from other literatures. Then questionnaire was prepared in English language. Before to actual data collection, data collectors were recruited based on their related professional background and then training was given on the data collection techniques in order to exercise data collection tools. Then pre-teste was done on $5 \%$ of hospital NICU patient files.

\subsection{Study Variables}

\subsubsection{Dependent Variable}

Neonatal mortality was the dependent variable.

\subsubsection{Independent Variable}

The independent variables included sex of the patient, address, age at admission, and gestational age, feeding, birth weight, and maternal age, and parity, antenatal care (ANC) follow-up place of delivery, place of birth.

\subsection{Operational Definition}

Neonate: a newborn baby less than 28 days of life.

Neonatal mortality: the death of a neonate that happens after birth but before 28 days of life.

Hypothermia: Any low body temperature measurement $\left(<36^{\circ} \mathrm{C}\right)$ diagnosed and recorded on charts during admission of neonates.

Premature: Any viable neonate before term $(<37$ weeks of gestation) that was already diagnosed by professionals in charge on admission of neonate to neonatal intensive care units.

Sepsis: Record of infection or sepsis diagnosed either clinically or with culture by professionals during admission of the neonate and as possible causes of death and designated or recorded on chart.

\subsection{Data Processing and Analysis}

The Collected data were cleaned, coded and entered into Epidata 3.1 and exported, to SPSS for windows version 23.0 for cleaning and analyses. Cross tabulation was done to see the distribution of cases and controls. The binary logistic regression model was used to test the association between dependent and independent variables. All variables with $\mathrm{P}$ value $<0.29$ in bivariate analysis were included in the multivariable analysis. Magnitude of association was measured by using an odds ratio at $95 \%$ confidence interval. Statistical significance was declared at $\mathrm{P}<0.05$. Finally, the data were presented with texts and tables and graphs.

\subsection{Ethical Consideration}

Ethical approval was obtained from the institutional review board of the Sheik Hassan Yabare Referral Hospital before conducting the research. Patient consent was not required for retrospective chart review study, as the institution reserves the right to possess the medical record of patients.

\section{Results}

\subsection{Socio-demographic Characteristics of Cases and Controls with Their Index Mothers}

In this study, a total of 76 neonates who died (cases) with their index mothers and 152 neonates who had survived (controls) with their index mothers were included making a response rate of $100 \%$. The mean $( \pm \mathrm{SD})$ age of mothers was $28.9 \pm 6.7$ years range from 17 to 44 years. Regarding to the residence $36(47.4 \%)$ of the cases and 51 (33.6\%) controls were living in rural areas. About sixty-three (82.9\%) of cases and $122(80.3 \%)$ of controls were aged $0-7$ days. Concerning to the sex of the neonates $42(55.3 \%)$ of the cases and 71 $(46.7 \%)$ of the controls were male. About $38(50 \%)$ of the cases and $49(32.2 \%)$ controls were preterm neonates. Regarding maternal parity $30(39.5 \%)$ of the cases and 39 (25.7\%) the controls were delivered their first born and the Birth type $69(90.8 \%)$ of the cases and $130(85.5 \%)$ of the controls were Singleton babies. (Table 1).

Table 1. Demographic characteristics of cases and controls admitted to the NICU Sheik Hassan Yabare Referral Hospital, East Ethiopia from July 30, 2017 to August 01, 2019.

\begin{tabular}{lllll}
\hline Variables & Category & Cases n=76 (\%) & Control n=152 (\%) & Total n=228 (\%) \\
\hline \multirow{2}{*}{ Residence } & Urban & $40(52.6)$ & $101(66.4)$ & $141(61.8)$ \\
& Rural & $36(47.4)$ & $51(33.6)$ & $87(38.2)$ \\
age of the neonate & 0-7days & $63(82.9)$ & $122(80.3)$ & $185(81.1)$ \\
& 8-28days & $13(17.1)$ & $30(19.7)$ & $43(18.9)$ \\
Sex of the neonate & Male & $42(55.3)$ & $71(46.7)$ & $113(49.6)$ \\
& female & $34(44.7)$ & $81(53.3)$ & $115(50.4)$ \\
Gestational age & Preterm & $38(50.0)$ & $49(32.2)$ & $87(38.2)$ \\
& Term & $35(46.1)$ & $96(63.2)$ & $131(57.5)$ \\
Birth type & Post term & $3(3.9)$ & $7(4.6)$ & $10(4.4)$ \\
\hline
\end{tabular}




\begin{tabular}{lllll}
\hline Variables & Category & Cases n=76 (\%) & Control $\mathbf{n}=\mathbf{1 5 2}(\mathbf{\%})$ & Total $\mathbf{n}=\mathbf{2 2 8}(\mathbf{\%})$ \\
\hline \multirow{3}{*}{ Parity } & Twins & $7(9.2)$ & $22(14.5)$ & $29(12.7)$ \\
& Primipara & $30(39.5)$ & $39(25.7)$ & $69(30.3)$ \\
\hline
\end{tabular}

Regarding Birth weight $36(47.4 \%)$ of cases and 46 (30.3\%) of controls had Birth weight less than 2500 grams and about 38 $(50 \%)$ of cases and $96(63.2 \%)$ of controls had Birth weight $2500-4000 \mathrm{gms}$.

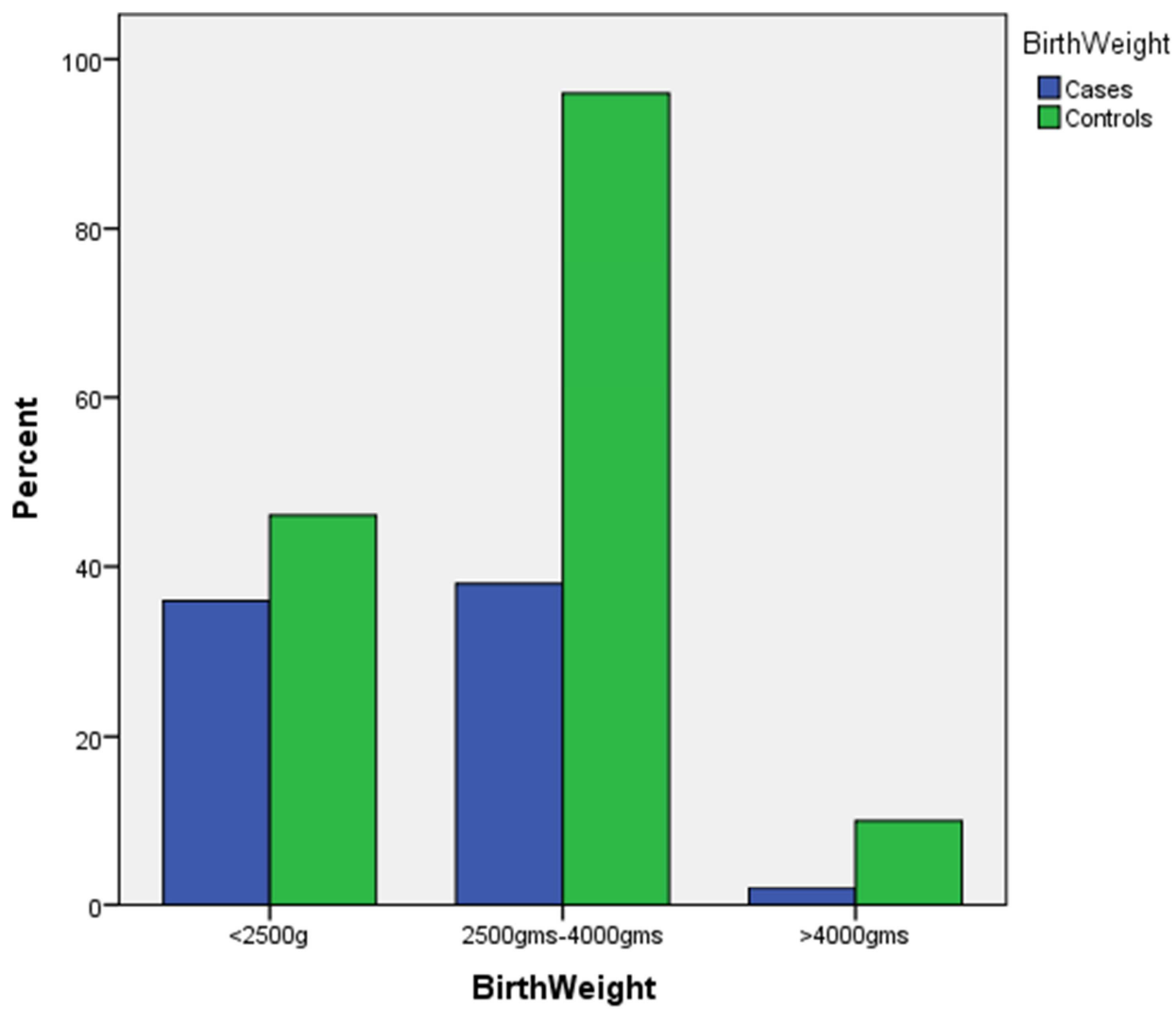

Figure 1. Birth weight of neonates admitted to the NICU at Sheik Hassan Yabare Referral Hospital, East Ethiopia from July 30,2017 to August 01, 2019.

\subsection{Characteristics of Neonates and Neonates Mothers in Bivariate and Multivariable Logistic Regression Analysis Result of the Study}

History of UTI/STI during the index pregnancy showed a statistically significant association with neonatal death. This study disclosed that, neonates born to mothers who had UTI/STI during the index pregnancy had 3.06 times higher odds of dying compared to those neonates born to mothers who did not have a UTI / STI during the index pregnancy [AOR=3.058; 95\% CI (1.136-8.229)]. (Table 2)

Table 2. Bivariate and multivariable logistic regression analysis result of the study of cases and controls admitted to the NICU Sheik Hassan Yabare Referral Hospital, East Ethiopia from July 30, 2017 to August 01, 2019.

\begin{tabular}{|c|c|c|c|c|c|c|}
\hline Variables & Category & Cases $n=76(\%)$ & Control $n=152(\%)$ & COR [95\% CI] & AOR [95\% CI] & P-Value \\
\hline \multirow[t]{3}{*}{ Residence } & Urban & $40(52.6)$ & $101(66.4)$ & $1.78(1.06-3.13)$ & $1.133(0.547-2.349)$ & 0.736 \\
\hline & Rural & $36(47.4 \%)$ & $51(33.6)$ & 1 & 1 & \\
\hline & $<2500 \mathrm{gms}$ & $36(47.4)$ & $46(30.3)$ & $0.256(.053-1.240)$ & $0.280(0.054-1.452)$ & 0.130 \\
\hline \multirow[t]{2}{*}{ Birth weight } & $2500-4000 \mathrm{gms}$ & $38(50.0)$ & $96(63.2)$ & $0.505(0.106-2.414)$ & $0.433(0.086-2.175)$ & 0.309 \\
\hline & $>4000 \mathrm{gms}$ & $2(2.6)$ & $10(6.6)$ & 1 & 1 & \\
\hline \multirow{2}{*}{ Parity } & Primipara & $30(39.5)$ & $39(25.7)$ & $0.529(0.294-.951)$ & $0.799(0.413-1.549)$ & 0.507 \\
\hline & Multipara & $46(60.5$ & $113(74.3$ & 1 & 1 & \\
\hline \multirow{2}{*}{ Antenatal visits } & Yes & $32(42.1)$ & $87(57.2)$ & $1.84(1.054-3.213)$ & $1.157(0.575-2.329)$ & 0.683 \\
\hline & No & $44(57.9)$ & $65(42.8)$ & 1 & & \\
\hline \multirow{2}{*}{$\begin{array}{l}\text { History of } \\
\text { neonatal fever }\end{array}$} & Yes & $52(68.4)$ & 7549.3) & $0.45(0.252-0.802)$ & $0.624(0.327-1.192)$ & 0.153 \\
\hline & No & $24(31.6)$ & $77(50.7)$ & 1 & 1 & \\
\hline \multirow{2}{*}{$\begin{array}{l}\text { Type of } \\
\text { breastfeeding }\end{array}$} & $\mathrm{EBF}$ & $51(67.1)$ & $129(84.9)$ & $2.749(1.432-5.28)$ & $1.728(0.790-3.778)$ & 0.171 \\
\hline & $\begin{array}{l}\text { Partial/bottle } \\
\text { feeding }\end{array}$ & $25(32.9)$ & $23(15.1)$ & 1 & 1 & \\
\hline
\end{tabular}




\begin{tabular}{|c|c|c|c|c|c|c|}
\hline Variables & Category & Cases n=76 (\%) & Control n=152 (\%) & COR $[95 \% \mathrm{CI}]$ & AOR $[95 \% \mathrm{CI}]$ & P-Value \\
\hline \multirow{2}{*}{ Maternal UTI } & Yes & $6(7.9)$ & $29(19.1)$ & $2.751(1.089-6.949)$ & $3.06(1.136-8.229)$ & $0.027^{*}$ \\
\hline & No & $70(92.1)$ & $123(80.9)$ & 1 & 1 & \\
\hline \multirow{2}{*}{ PROM } & Yes & $32(42.1)$ & $79(52)$ & $1.488(0.854-2.59)$ & $1.321(0.687-2.544)$ & 0.404 \\
\hline & No & $44(57.9)$ & $73(48)$ & 1 & 1 & \\
\hline \multirow{3}{*}{ Place of delivery } & Hospital & $58(76.3)$ & $129(84.9)$ & $2.002(0.77-5.19)$ & $1.730(0.544-5.499)$ & 0.353 \\
\hline & $\begin{array}{l}\text { health } \\
\text { centre/clinic }\end{array}$ & $9(11.8)$ & $13(8.6)$ & $1.3(0.38-4.48)$ & $1.423(0.350-5.791)$ & 0.623 \\
\hline & Home & $9(11.8)$ & $10(6.6)$ & 1 & 1 & \\
\hline \multirow{2}{*}{ IUGR } & Yes & $8(10.5)$ & $3(2.0)$ & $0.171(0.044-.665)$ & $0.248(0.057-1.088)$ & 0.065 \\
\hline & No & $68(89.5)$ & $149(98.0)$ & 1 & 1 & \\
\hline
\end{tabular}

$\mathrm{CI}=$ confidence interval $\mathrm{COR}=$ crude odd ratio $\mathrm{AOR}=$ adjusted odd ratio; $*$ Significant variable.

\section{Discussion}

The finding of this study showed that one maternal related factor had a significant effect on the risk of neonatal death. In this study, more than two third of the cases $(82.9 \%)$ were with early neonatal death $(<7$ days $)$ which is more or less comparable with the studies conducted previously in Jimma (69.1\%), Indonesia (75.6\%) and (88.7\%) Tunisia $[9,25,26]$. The reason could be mainstream of neonatal mortality in low and middle-income countries are related to circumstances of labor, intra-partum and the immediate newborn resuscitation practices.

The result of this study disclosed that neonates born to mothers who had urinary tract infection/ Sexual transmitted infection during the index pregnancy had 3.06 times higher odds of dying compared to those neonates born to mothers who did not had a urinary tract infection during the index pregnancy $[\mathrm{AOR}=3.058 ; 95 \%$ CI $(1.136-8.229)]$. This finding agreed by study conducted in Nigeria showed that the maternal illness including urinary tract infection was associated with neonatal deaths [27]. This encounter is more or less comparable with the findings of study conducted in Pakistan showed that maternal urinary tract infection was risk factor for neonatal death [28]. This findings may support for the reason that maternal urinary tract infection is often associated with neonatal death, especially if untreated during pregnancy or labor, it may results the growth of the maternal body by the infectious agent which the mother transfers to fetus then due to physiological transition makes fetus/neonate un enable to defend itself after delivery which subsequently causes death.

This finding is contrary with a study conducted in Kenya which did not detected an association between neonatal mortality and STIs or any other distinctive [29]. The discrepancy could be explained due to study design difference and the study setting where data was gathered, or difference between protocols being used in these hospitals for prevention of infection on pregnant women during pregnancy intrapartum and postpartum period.

Among patients from a total of cases and controls the presence of risk factors such as Residence, Birth weight, Parity, Antenatal visits, Type of breastfeeding, premature rupture of membranes or Place of delivery, Intrauterine growth retardation were not detected to be risk factors for neonatal death.

\section{Conclusion and Recommendations}

In conclusion, this study has found that one maternal had contributed to the risk of neonatal death. History of maternal Urinary Tract Infection was identified as possible independent risk factor of neonatal demise. On the other hand; Residence, Birth weight, Parity, Antenatal visits, Type of breastfeeding, premature rupture of membranes or Place of delivery, Intrauterine growth retardation were not found to be statistically associated with risk of neonatal death.

Based on the finding it is recommended that need for lowcost and accurate methods diagnostic screening and treatment for maternal Urinary Tract Infection during pregnancy as well as institutional delivery to reduce newborn deaths.

\section{Limitation of the Study}

Since the study is done on admitted neonates, therefore results might shortfall generalizability to the whole population in the catchment area.

\section{Abbreviations and Acronyms}

EDHS Ethiopia Demographic and Health Survey

NICU Neonatal intensive care unit

UTI Urinary Tract Infection

EBF Exclusive breast feeding

SDG Sustainable Development Goals

SPSS Statistical Package for Social science

SHYRH Sheik Hassan Yabare Referral Hospital

\section{Acknowledgements}

We would like to extend our appreciation Sheik Hassan Yabare Referral Hospital for providing the ethical clearance to conduct the study in the facility.

We would also like express our gratitude and sincerity to the data collectors for their modest cooperation and hardworking.

\section{References}

[1] Organization, W. H., Neonatal and perinatal mortality: country, regional and global estimates. 2006. 
[2] Pathirana, J., et al., Neonatal death: Case definition \& guidelines for data collection, analysis, and presentation of immunization safety data. 2016. 34 (49): p. 6027-6037.

[3] UNICEF, WHO, The World Bank, United Nations. Levels and trends in child mortality: report 2014. New York, USA: UNICEF, 2014. 2016.

[4] Devine, S. and G. Taylor, Every Child Alive: The urgent need to end newborn deaths. 2018: Unicef.

[5] Yaya, Y., et al., Maternal and neonatal mortality in south-west Ethiopia: estimates and socio-economic inequality. 2014. 9 (4): p. e96294.

[6] Kebede, B., et al., Prevalence and associated factors of neonatal mortality in North Gondar Zone, Northwest Ethiopia. 2012. 26 (2): p. 66-71.

[7] Annan, G. N. and Y. J. A. i. P. H. Asiedu, Predictors of Neonatal Deaths in Ashanti Region of Ghana: A CrossSectional Study. 2018. 2018.

[8] Akinyemi, J. O., E. A. Bamgboye, and O. J. B. p. Ayeni, Trends in neonatal mortality in Nigeria and effects of biodemographic and maternal characteristics. 2015. 15 (1): p. 36.

[9] Titaley, C. R., et al., Determinants of neonatal mortality in Indonesia. 2008. 8 (1): p. 232.

[10] Wolde, H. F., et al., Factors affecting neonatal mortality in the general population: evidence from the 2016 Ethiopian Demographic and Health Survey (EDHS)-multilevel analysis. 2019. 12 (1): p. 610.

[11] Arafa, M. A. and M. A. J. S. m. j. Alshehri, Predictors of neonatal mortality in the intensive care unit in Abha, Saudi Arabia. 2003. 24 (12): p. 1374-1376.

[12] Reyes, J., et al., Neonatal mortality and associated factors in newborn infants admitted to a Neonatal Care Unit. 2018. 116 (1): p. $42-48$

[13] Owusu, B. A., et al., Neonatal mortality at the neonatal unit: the situation at a teaching hospital in Ghana. 2018. 18 (2): p. 369-377.

[14] Garg, P. and S. Gogia, Reducing neonatal mortality in developing countries: low-cost interventions are the key determinants. Journal of Perinatology, 2009. 29 (1): p. 74.

[15] Rarani, M. A., et al., Changes in socio-economic inequality in neonatal mortality in Iran between 1995-2000 and 2005-2010: an Oaxaca decomposition analysis. 2017. 6 (4): p. 219.
[16] Oestergaard, M. Z., et al., Neonatal mortality levels for 193 countries in 2009 with trends since 1990: a systematic analysis of progress, projections, and priorities. 2011. 8 (8): p. e1001080

[17] Organization, W. H., Every newborn: an action plan to end preventable deaths. 2014.

[18] Blencowe, H., et al., Tetanus toxoid immunization to reduce mortality from neonatal tetanus. 2010. 39 (suppl 1): p. i102i109.

[19] UNICEF. Neonatal mortality 2019 [cited 2019 29/12/2019]; Available from: https://data.unicef.org/topic/childsurvival/neonatal-mortality/.

[20] Liu, L., et al., Global, regional, and national causes of child mortality in 2000-13, with projections to inform post-2015 priorities: an updated systematic analysis. Lancet, 2015. 385 (9966): p. 430-40.

[21] Estimation, U. N. I.-a. G. f. C. M., et al., Levels \& Trends in Child Mortality: Report 2018, Estimates Developed by the. 2018: United Nations Children's Fund.

[22] Ethiopian Public Health Institute (EPHI), I., Mini Demographic and Health Survey, C. S. Agency, Editor. 2019, Rockville, Maryland, USA: EPHI, ICF: Addis Ababa.

[23] CSA, Population and Housing Census of Ethiopia, C. S. Agency, Editor. 2015: Addis Ababa.

[24] Kolobo, H. A., T. E. Chaka, and R. T. J. J. o. C. N. Kassa, Determinants of neonatal mortality among newborns admitted to neonatal intensive care unit Adama, Ethiopia: A casecontrol study. 2019.8 (4): p. 232.

[25] Debelew, G. T., M. F. Afework, and A. W. J. P. o. Yalew, Determinants and causes of neonatal mortality in Jimma zone, southwest Ethiopia: a multilevel analysis of prospective follow up study. 2014. 9 (9).

[26] Ben, E. H. N., et al., Determinants of neonatal mortality in a Tunisian population. 2010.88 (1): p. 42-45.

[27] Adetola, A. O., et al., Neonatal mortality in an urban population in Ibadan, Nigeria. 2011. 52 (5): p. 243-250.

[28] Kousar, T., et al., Risk factors and causes of death in Neonates 2010. 35 (2): p. 205-208.

[29] Warr, A. J., et al., Sexually transmitted infections during pregnancy and subsequent risk of stillbirth and infant mortality in Kenya: a prospective study. 2019. 95 (1): p. 60-66. 